\title{
Bestimmung des stochastischen Zeitverhaltens in Supply Chains
}

\author{
DR.-ING. INGOLF MEINHARDT, \\ M.ENG. FELISIA SUNARJO, \\ Prof. DR.-ING. HABIL. HANS-GEORG MARQUARDT \\ INSTITUT FÜR FÖRDERTECHNIK, BAUMASCHINEN UND LOGISTIK, TECHNISCHE UNIVERSITÄT DRESDEN
}

Stochastische Einflüsse wirken auf alle elementaren Prozesse einer Lieferkette. Sie äußern sich in variablen Fertigungs-, Transport- oder Durchlaufzeiten sowie Lagerbeständen zur Prozessentkopplung. Die Auswirkungen auf Liefertreue, Vorlaufzeiten, Bestände oder Kosten für die gesamte Supply Chain sind zurzeit nur simulativ abschätzbar. Das hier vorgestellte numerische Verfahren kann mit geringem Aufwand ähnliche, statistisch abgesicherte Kennzahlen für das Zeitverhalten liefern.

Stochastic influence has significant impact on all elementary processes of a supply chain. This impact is reflected on variable production, transportation or lead time as well as the inventory of decoupling process. At present, the implication of delivery reliability, lead time, inventory or total cost for the whole supply chain can only be evaluated by using simulation. This work proposes a less time- and cost-consuming numerical approach to provide the reliable statistical parameters for the time behaviour.

\section{Einführung}

Sowohl in der Planungsphase als auch beim Betrieb von Supply Chains entsteht immer wieder die Frage nach zu erwartenden Leistungskennzahlen, richtiger Prozessauslegung und ausreichender Dimensionierung der Elemente. Gewachsene Komplexität und Intransparenz verschiedenster Einflüsse auf die Supply Chain führen jedoch zu einer zunehmenden Unsicherheit über den Prozessverlauf.

Bewertungen zum Leistungsverhalten sind in Supply Chain Management (SCM)-Software-Systemen zwar über betriebswirtschaftliche und/oder technisch orientierte Kennzahlensysteme möglich, aber stets nachträglich auf Grund erfasster Prozessdaten (z. B. als Nachkalkulation). Auch das Supply Chain Operations Reference Model (SCOR) sieht nicht vor, Kennzahlen im prognostischen bzw. planerischen Sinne auf Grund der Lieferkettenstruktur, des Kundenverhaltens oder der aktuellen Auftragslage zu bestimmen.

In [Erengüc99] wird als Ziel weiterer Forschungen u.a. die Analyse von Supply Chains hinsichtlich der Quantifizierung von Produktionskapazitäten, Durchlaufzeiten und Lagerbeständen (bei Berücksichtigung der Unsicherheit/Stochastik im zeitlichen Verlauf) formuliert. Gleichzeitig orientiert sich eine übergreifende Betrachtung auf drei Hauptabschnitte (Beschaffen, Herstellen, Liefern) .

Obwohl viele Forschungsthemen die Vernetzung in der Supply Chain und die Integration von einzelnen Organisationen betreffen, stecken Erkenntnisse zur Prozessausrichtung und fortgeschrittenen Planung über Unternehmensgrenzen hinweg noch in den Kinderschuhen [Stadtler05], [Croom00]. So legt z. B. Raghavan ein analytisches Model auf der Basis eines offenen allgemeinen Bediennetzwerkes vor, um die mittlere Durchlaufzeit als eine kritische Leistungskennzahl in einer „make to order“- Supply Chain zu ermitteln [Raghavan01]. Da es aber keine exakte Lösung für die Berechnung eines allgemeinen Bediennetzwerkes gibt, wird eine Approximation vorgenommen.

Die fehlenden Kenntnisse bzw. Lösungsmethoden haben dazu geführt, dass als gebräuchliches Werkzeug zur Bewertung komplexer, vernetzter Systeme einzig die Simulation angesehen wird. Darunter versteht man „das Nachbilden eines Systems mit seinen dynamischen Prozessen in einem experimentierbaren Modell, um zu Erkenntnissen zu gelangen, die auf die Wirklichkeit übertragbar sind. Insbesondere werden die Prozesse über die Zeit entwickelt" [VDI 3633].

Der Anwendung simulativer Verfahren sind durch Rechentechnik und leistungsfähige Simulatoren nahezu keine Grenzen mehr gesetzt. Dennoch stellt sich die Entwicklung von Simulationsmodellen für konkrete Anwendungen in aller Regel als aufwändiges, langwieriges und teures Verfahren heraus, das den Einsatz von Simulationsexperten erfordert. Da ein Simulationslauf meist von vielen Parametern beeinflusst wird, sind viele Simulationsläufe und umfangreiche Auswertungen notwendig. Gerade in frühen Planungsphasen aber sind viele Einflussgrößen noch nicht fixiert, so dass mittels Simulation nur bedingt funktionale Aussagen über den Einfluss einzelner Parameter auf das Simulationsergebnis möglich sind. Außerdem ergeben sich häufig auch strukturelle Änderungen, die einen erheblichen Änderungsaufwand am Simulationsmodell nach sich ziehen. 
Auf dem Gebiet des Projektmanagements sind analytische Verfahren zur Planung, Steuerung und Kontrolle des Projektablaufes hinsichtlich der Termine, Kapazitäten und Kosten bekannt. Eine Übertragung des Vorgehens in diesen als Netzplantechnik bezeichneten Verfahren auf die Belange der Supply Chains erscheint sinnvoll auf Grund naheliegender Analogien.

Nach [DIN 69900] ist ein Netzplan „die graphische Darstellung von Ablaufstrukturen, die die logische und zeitliche Aufeinanderfolge von Vorgängen veranschaulichen“.

Dabei werden folgende grundlegende Annahmen getroffen:

- Ein Projekt kann in einzelne Aktivitäten zerlegt werden.

- Jede Aktivität hat ein Start- und ein Endereignis.

- Die Zeitspanne jeder einzelnen Aktivität ist bekannt oder kann geschätzt werden.

Die unterschiedlichen Netzplantechniken wie Critical Path Method (CPM), Program Evaluation and Review Technique (PERT) oder Graphical Evaluation and Review Technique (GERT) sind aber nur bedingt geeignet, die Problemstellungen von Supply Chains zu lösen. Entweder ist keine Abbildung bestimmter Prozessstrukturen (PERT: fehlende Abbildung für alternative und rekursive Vorgänge) oder keine vollständige analytische Lösung möglich (GERT) [Malcolm59], [Neumann75].

Des Weiteren führen die Annahmen, die diesen Verfahren zu Grunde liegen, zu systematischen Fehlern. Sie liefern zu optimistische Schätzungen der tatsächlichen Projektdauer bei zu geringer Genauigkeit. Die Ursachen liegen in der vereinfachten Ermittlung der Parameter für die Verteilungsfunktion der Zeitdauer auf Grund dreier Zeitschätzwerte:

- optimistische, pessimistische und häufigste Zeitdauer,

- der Fixierung allein auf eine beta-verteilte Aktivitätsdauer,

in der Annahme, dass das Netz nur einen einzigen dominanten „kritischen Pfad“ besitzt. Sobald es aber mehrere konkurrierende Pfade gibt, kann auf Grund der Stochastik jeder dieser Pfade die gesamte Projektdauer erheblich beeinflussen. Diese Option bleibt in PERT durch die Reduzierung der Betrachtung auf die Summe der Mittelwerte (und nach dem zentralen Grenzwertsatz der Annahme einer normalverteilten Gesamtdauer) unberücksichtigt [Ioannou98].

\section{Numerisches Verfahren zur Berechnung sto-chastischer Vorgänge}

Die Defizite in den Verfahren der Netzplantechnik und die Unzulänglichkeiten der Simulation waren Anlass zur Suche nach neuen Lösungsmöglichkeiten für die Ermittlung des Zeitverhaltens innerhalb von Netzwerken. Im Ergebnis entstand ein Modell, bei dem zunächst die Vorgehensweise von PERT beibehalten wird: Die Vorgänge im Netzplan sind durch Anfangs- und Endereignisse in der Weise verknüpft, dass stets alle vorgelagerten Vorgänge abgeschlossen sein müssen und alle nachfolgenden Vorgänge zeitsynchron beginnen (deterministische Prozessausführung). In einer zweistufigen Berechnung werden die frühesten und spätesten Starttermine für jedes Ereignis (Vorwärts- bzw. Rückwärtsrechnung) und daraus mögliche Puffer- oder Reservezeiten sowie der kritische Weg bestimmt.

Das entwickelte numerische Verfahren liefert durch Diskretisierung der Zeitverteilungen aller Vorgangsdauern und deren operationsbezogenen statistischen Verknüpfungen Aussagen über das Netzwerk. Im Unterschied zu den traditionellen Netzplanverfahren erfolgt aber die Berechnung nicht mehr auf der Basis statistischer Momente (Mittelwert, Varianz), sondern durch Berechnung von Dichte- und/oder Verteilungsfunktionen, aus denen schließlich die gesuchten Momente ermittelt werden. Genau wie die Vorgänge selbst in unterschiedlicher Weise voneinander abhängen können, müssen auch die Verknüpfungen der zugehörigen Funktionen entsprechend dieser Abhängigkeit (Operation) gebildet werden.

Neben der verbesserten Genauigkeit der Ergebnisse bietet sich dabei gleichzeitig die Möglichkeit, auch andere als die in PERT ausschließlich benutzten Beta-Verteilungen für die Vorgangsdauern zu Grunde zu legen.

\subsection{Verfahren bei deterministischer Prozessstruktur}

\subsubsection{Vorgangssequenzen}

Sei die Dauer eines Vorgangs k eine Zufallsgröße $T_{k}$ mit der Verteilungsfunktion $F_{k}(x)$ und der Dichtefunktion $f$ $\mathrm{k}(\mathrm{x})$. Dann gilt

$\mathrm{F}_{\mathbf{k}}(\mathrm{x})=\mathrm{P}\left(\mathrm{T}_{\mathbf{k}} \leq \mathrm{x}\right)$

Für zwei nacheinander auszuführende (sequentielle) Vorgänge 1 und 2 (Abb. 1, links) ergibt sich demnach eine zufällige Gesamtdauer $\mathrm{T}_{\mathrm{G}}=\mathrm{T}_{1}+\mathrm{T}_{2}$. Die Summe zweier unabhängiger und diskret verteilter Zufallsgrößen kann mittels diskreter Faltung ihrer Dichtefunktionen bestimmt werden: 


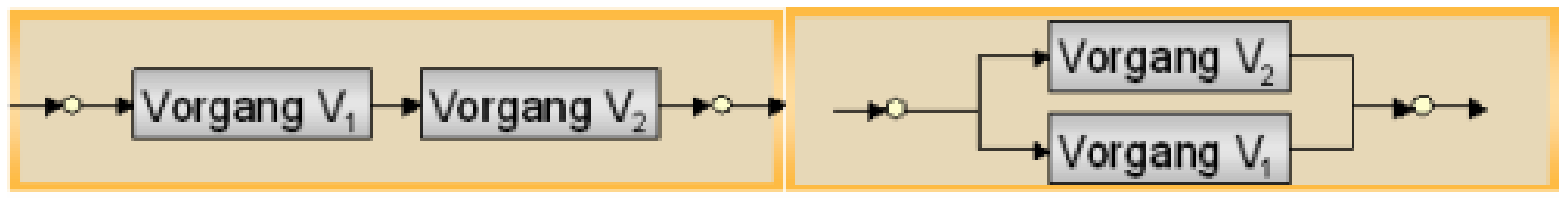

Abbildung 1: Deterministische Prozessstruktur: Sequentielle und parallele Vorgänge

$$
f_{1}(x)+f_{2}(x)=f_{1+2}(x)=\sum_{\mathbf{z}=\mathbf{1}}^{h}\left(f_{1}(x-y) \cdot f_{2}(y)\right)
$$

Die Summationsgrenzen a und b begrenzen den möglichen Definitionsbereich und werden als Summe der unteren bzw. oberen Grenzwerte der Vorgangsdauern bestimmt. ${ }^{1}$

Die Faltungsoperation ist kommutativ und assoziativ, durch wiederholte Anwendung der Faltung kann demnach für mehrere unabhängige, sequentielle Vorgänge die Gesamtzeitverteilung berechnet werden.

\subsubsection{Zeitlich parallele Vorgänge}

Die Annahme von zwei oder mehreren parallelen (im Sinne von zeitgleich beginnenden) Aktivitäten zufälliger Dauer (Abb. 1, rechts) erlaubt keine sichere Aussage, welche Aktivität im Einzelfall länger dauert und inwiefern dadurch der kritische Pfad (und damit die Gesamtdauer) beeinflusst wird.

Mit dem Ende der Parallelität ist eine Synchronisation erforderlich, d.h. es muss bis zum Abschluss des letzten parallelen Vorgangs gewartet werden. Das muss jedoch nicht automatisch der Vorgang mit der größten mittleren Dauer sein, sondern kann auch ein im Mittel nicht kritischer, aber zeitlich stark variierender Vorgang sein.

Für die Ermittlung der Gesamtzeitverteilung ist also jeweils das Maximum der zufälligen Zeitdauern $\tau_{1}, \tau_{2}$, bis $\tau_{\mathrm{k}} \mathrm{zu}$ bestimmen.

$\tau_{\mathrm{t}}=\max \left(\tau_{1} ; \tau_{\mathbf{g}} ; \ldots ; \tau_{\mathbf{k}}\right)$

Im statistischen Sinne ist dies gleichbedeutend mit dem Produkt der Verteilungsfunktionen aus den Vorgangsdauern.

$F\left(\tau_{G}\right)=F_{1}(x) \cdot F_{1}(x) \cdot \ldots \cdot F_{1}(x)=\prod_{i=1}^{1} F_{i}(x)$

Das Ergebnis ist eine „Maximum“-Verteilung mit folgenden Eigenschaften:

- Untere und obere Grenze des Definitionsbereichs sind die jeweiligen Maxima der unteren bzw. oberen Grenzwerte der Vorgangsdauern.

- Der Mittelwert ist größer als jeder der zu Grunde liegenden Verteilungen.

- Die Varianz ist kleiner als die größte Varianz der Einzelvorgänge.

Eine Vergrößerung der mittleren Gesamtdauer bei parallel ablaufenden Vorgängen ergibt sich also unabhängig von Auslastung, Bedienstrategie o.Ä. bereits aus der Struktur des Netzplans und der Verteilung der Zeiten. Sie äußert sich in einer zusätzlichen, strukturbedingten Wartezeit am Ende eines jeden Vorgangs. Die Größe dieser strukturbedingten Wartezeit wird maßgeblich durch die Varianz der Vorgangsdauern bestimmt. Sie kann ermittelt werden aus der Differenz der mittleren Gesamtdauer und dem Maximum der mittleren Dauern der parallelen Vorgänge.

$E\left(\tau_{\nabla}\right)=E\left(\tau_{\mathbf{g}}\right)-\max \left(E\left(\tau_{1}\right) E\left(\tau_{3}\right), \ldots, E\left(\tau_{n}\right)\right)$

Praktisch bedeutet diese strukturbedingte Wartezeit oft eine Verlängerung der Lagerverweilzeit und damit eine Bestandserhöhung.

\subsection{Verfahren bei stochasticher Prozessstruktur}

Während das im vorigen Abschnitt vorgestellte Verfahren nur für deterministische Prozessausführung anwendbar ist, sind in der Praxis häufig auch stochastische Prozessausführungen anzutreffen. So wird z. B. nach einer Qualitätskontrolle nur ein Teil der Erzeugnisse an den nachfolgenden Prozess übergeben, während fehlerhafte Produkte in einem anderen Prozess nachbearbeitet werden (Alternative) und erneut zur Qualitätskontrolle kommen (Rekursion).

\footnotetext{
1 Stetige Verteilungsfunktionen sind oftmals nicht oder allenfalls einseitig begrenzt. Mit der Diskretisierung kann aber ein Genauigkeitsmaß in Form der Quantile eingeführt werden. So ist es im allgemeinen ausreichend, die untere und obere Grenze festzulegen mit $\mathrm{P}(\mathrm{X} \leq \mathrm{a})<0,0001$ bzw. $\mathrm{P}(\mathrm{X} \leq \mathrm{b})<0,9999$. Bei normalverteilten Zeitdauern mit dem Mittelwert $\mu$ und der Standardabweichung $\sigma$ gilt dann näherungsweise $\mathrm{a}=\mu-4 \sigma$ bzw. $\mathrm{b}=\mu+4 \sigma$
} 
Es ergeben sich für einzelne Vorgänge unterschiedliche Ausführungswahrscheinlichkeiten und damit ein unterschiedlich gewichteter Einfluss der Vorgangsdauer auf die Gesamtdurchlaufzeit.

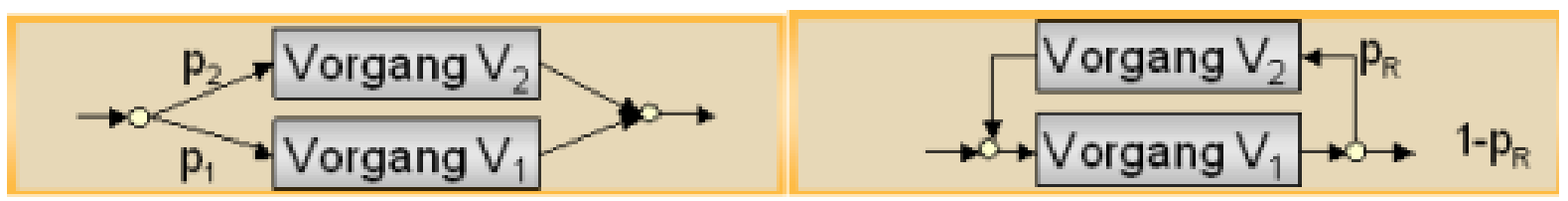

Abbildung 2: Stochastische Prozessstruktur: Alternative und rekursive Vorgänge

\subsubsection{Alternative Prozessausführung}

Alternative Prozessausführung bedeutet das Vorhandensein von zwei oder mehreren möglichen Fortsetzungen der Prozessdurchführung, von denen genau eine realisiert wird. Dabei ist die Kenntnis des Entscheidungskriteriums unbedeutend, es interessieren lediglich die Ausführwahrscheinlichkeiten $p_{i}$ der möglichen Alternativen.

Die Gesamtzeit kann als Summe der mit $\mathrm{p}_{\mathrm{i}}$ gewichteten Dichte der Vorgangsdauern berechnet werden:

$f\left(\tau_{w}\right)=\sum_{i=1}^{n}\left(p_{i} \cdot f_{i}(x)\right) \quad$ mit $\sum_{i=1}^{n} p_{i}=1$

Die Berechnung der Summe ist wie bei den Vorgangssequenzen als diskrete Faltung vorzunehmen. Im Unterschied zu letzterer werden aber die prozentualen Häufigkeiten des Auftretens (Dichte) mit Hilfe der Ausführwahrscheinlichkeit gewichtet.

Für den Definitionsbereich und die Momente einer solchen „Alternativ“-Verteilung können folgende generelle Aussagen abgeleitet werden:

- Untere und obere Grenze des Definitionsbereichs ergeben sich als Minimum der unteren bzw. Maximum der oberen Grenzwerte der Vorgangsdauern.

- Der Mittelwert liegt zwischen dem kleinsten und größten Mittelwert der zu Grunde liegenden Verteilungen.

- Die Varianz wird umso größer, je weiter die mittleren Vorgangsdauern auseinander liegen.

\subsubsection{Rekursive Prozessausführung}

Rekursive Prozessausführung bedeutet das wiederholte Ausführen von Vorgängen auf Grund eines hier nicht näher spezifizierten Kriteriums (mehrphasige Bearbeitung, Reklamation o. Ä.). Von der Funktion her ähnelt es der im vorigen Abschnitt beschrieben Alternative. Nach dem Ende von Vorgang $V_{1}$ (hier als Kontrollprozess bezeichnet) gibt es wahlweise zwei mögliche Fortsetzungen:

- Mit der Wahrscheinlichkeit $0<p_{\text {rek }}<1$ wird als nächstes Vorgang $V_{2}$ (Nacharbeit) ausgeführt.

- Dementsprechend ist (1- $\left.\mathrm{p}_{\mathrm{rek}}\right)$ die Wahrscheinlichkeit für die Fortsetzung mit Vorgang $\mathrm{V}_{3}$

Im Unterschied zur alternativen Prozessausführung wird aber nach Abschluss von $V_{2}$ erneut $V_{1}$ (rekursiv) ausgeführt und der Entscheidungspunkt durchlaufen. Es kommt damit zur wiederholten, zyklischen Ausführung von Vorgängen und dadurch zu einer wesentlichen Erhöhung des Zeitbedarfs. Die erwartete Anzahl der Aktivierungen eines Vorgangs heißt Aktivierungszahl.

Dessen Größe wird in entscheidender Weise von der Reklamationsquote $p_{\text {rek }}$ (Wahrscheinlichkeit für Mängel, die eine Nacharbeit erfordern) bestimmt. Es ergeben sich damit die Aktivierungszahlen für die beiden Vorgänge $\mathrm{zu}$

$$
\begin{aligned}
& q_{\mathbf{k}, \mathbf{y}}=\sum_{\mathbf{l}=\mathbf{0}}^{\infty} \mathrm{p}_{\mathbf{m}} \mathbf{l}=\frac{1}{1-\mathrm{p}_{\mathbf{m}}} \\
& \mathrm{q}_{\mathbf{M} \mathbf{b}, \mathbf{M}}=\sum_{\mathbf{l}=\mathbf{l}} \mathrm{p}_{\mathbf{m}} \mathbf{l}=\frac{1}{1-\mathrm{p}_{\mathbf{n} \mathbf{l}}}-1
\end{aligned}
$$




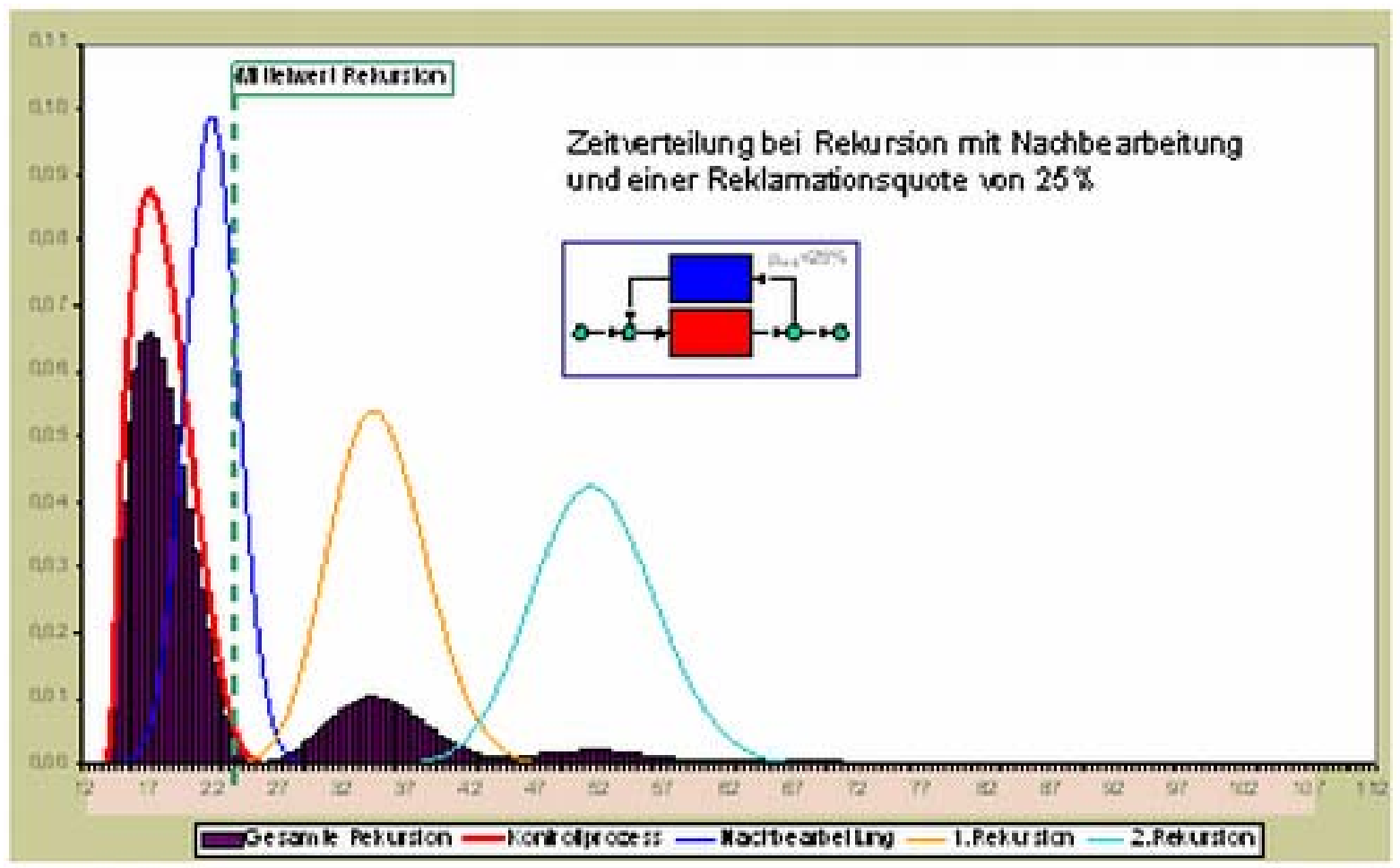

Abbildung 3: Entwicklung der Durchlaufzeitverteilung bei Rekursion

Theoretisch werden unendlich viele Rekursionen zugelassen, ihr Anteil hat sich aber bei gängigen Reklamationsquoten $\mathrm{p}_{\mathrm{rek}}<20 \%$ bereits nach wenigen Durchläufen auf weniger als $0,1 \%$ reduziert und ist damit vernachlässigbar. Für die Berechnung bedeutet dies die Beschränkung auf eine konfigurierbare Genauigkeit, die im Allgemeinen nach 3 bis 5 Rekursionen erreicht wird.. Auch in der Praxis ist es üblich, nach einer begrenzten Anzahl von Rekursionsdurchläufen jegliche Nacharbeit aus Qualitäts- oder Kostengründen einzustellen.

Die Berechnung der Zeitverteilung erfolgt aus einer Kombination der bereits vorgestellten Berechnungen für alternative und sequentielle Vorgänge.

$f\left(\tau_{\mathbf{g}}\right)=\left(1-p_{\mathbf{m}}\right) \cdot f\left(\tau_{1}\right)+\sum_{\mathbf{l}=1}\left(p_{\mathbf{m}}{ }^{1} \cdot\left(f\left(\tau_{1}\right)+f\left(\tau_{1}\right)\right)\right)$

Definitionsbereich und Momente der Verteilungsfunktion für Rekursionen lassen folgende verallgemeinerbare Aussagen zu:

- Untere Grenze des Definitionsbereichs ist der untere Grenzwert des Kontrollprozesses.

- Die obere Grenze hängt ab von den oberen Grenzwerten von Kontroll- und Nachbearbeitungsprozess und der Anzahl der Rekursionen. Die wiederum wird durch die Reklamationsquote bestimmt.

- Der Mittelwert ergibt sich zu

$$
E\left(\tau_{\mathbf{G}}\right)=q_{\mathbf{M t}, \mathbf{Y}} \cdot E\left(\tau_{1}\right)+q_{\mathbf{M t}, \mathbf{n}} \cdot E\left(\tau_{\mathbf{1}}\right)
$$

- Auf Grund des großen Definitionsbereichs sind die Abweichungen vom Mittelwert und damit die Varianz recht groß. Die Varianz wird maßgeblich von der Anzahl der Rekursionen, also der Reklamationsquote bestimmt und kann nur aus der resultierenden Verteilungsfunktion ermittelt werden.

\section{Berechnungswerkzeug}

Die vorgestellten Berechnungsverfahren wurden in einem Anwendungsprogramm (Abb. 4 und 5) implementiert. Es ermöglicht

- das Erstellen einer beliebigen Prozessstruktur,

- die Parametrierung der Einzelvorgänge auf Grund einer Reihe implementierter statistischer Verteilungen

- die Berechnung der Zeitverteilungen für alle Start- und Endereignisse,

- die Visualisierung der Ergebnisse: Histogramm der Zeitverteilungen, Wahrscheinlichkeiten für Termineinhaltung, strukturbedingte Wartezeiten, Zeitdauern als Gantt-Diagramm,

- Übergabe der Berechnungsergebnisse an andere Programme. 


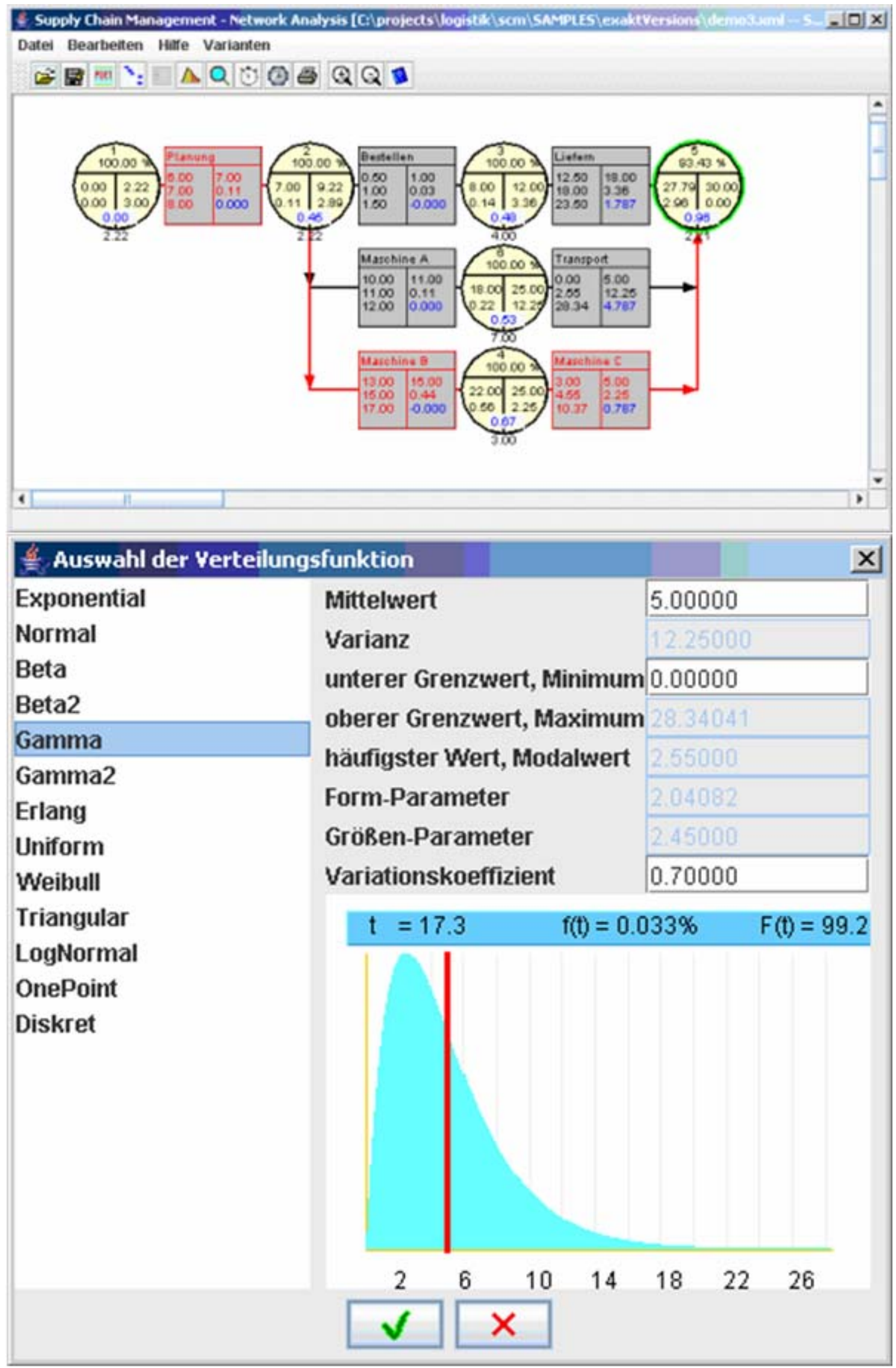

Abbildung 4: Screenshot der Nutzeroberfläche (oben) und Dialog zur Definition Vorgangsdauern (unten) 


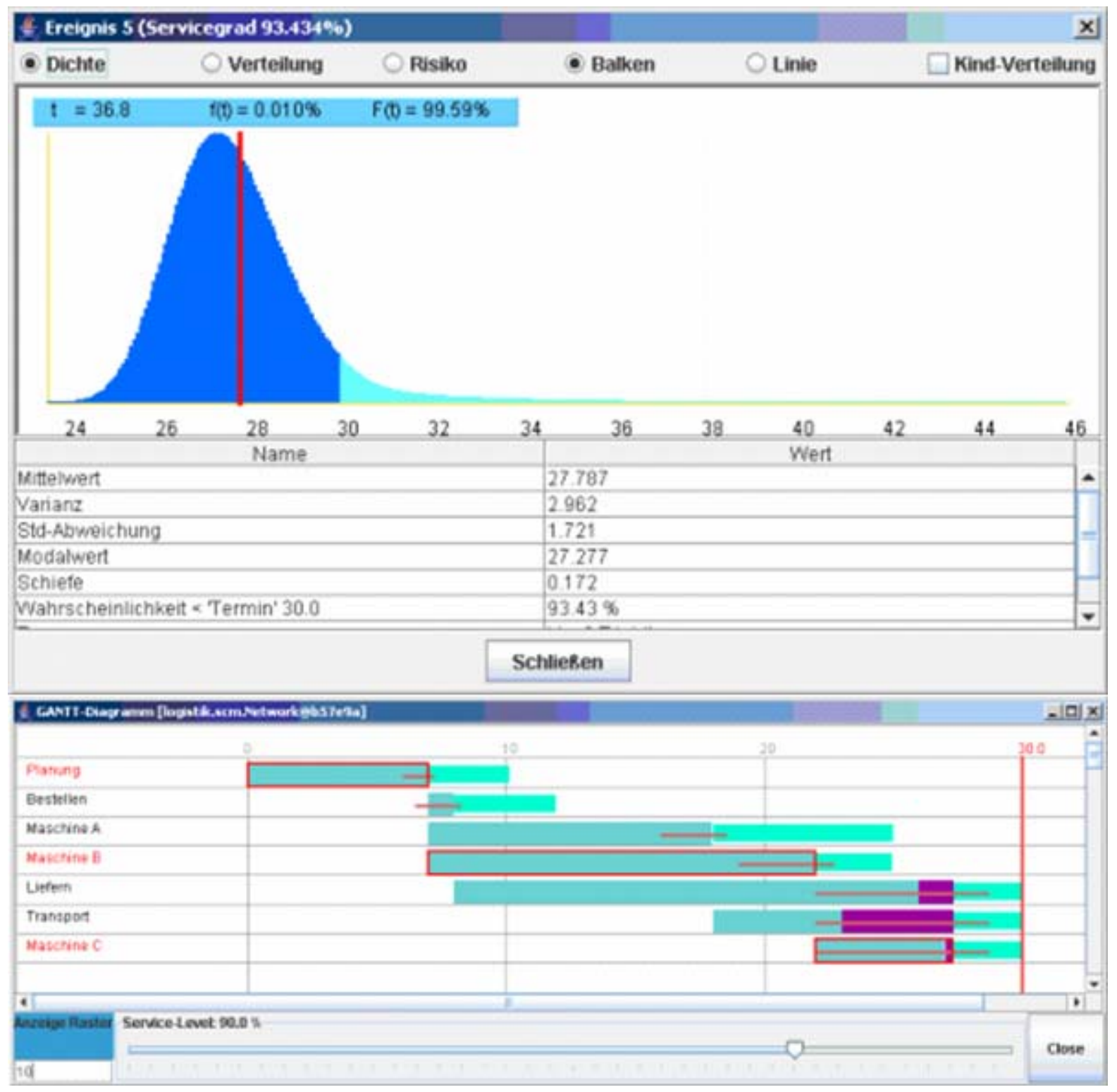

Abbildung 5: Screenshot Berechnungsergebnisse:

Oben - Verteilung Gesamt-Durchlaufzeit und Termineinhaltung (verspätete Lieferung bei $t>30$ ), Unten - Gantt-Diagramm

\section{Anwendungsbeispiel}

Für ein Zuliefernetzwerk in der Automobilindustrie wurde das Durchlaufzeitverhalten untersucht. Die Struktur des Netzwerkes ist vereinfacht in Abbildung 6 dargestellt.

Die internen Abläufe bei den einzelnen Zulieferer wurden jeweils zusammengefasst zu einem Vorgang. Für die Vorgangsdauern wurden Zeitbedarfe ausgewertet und in Form von Zeitschätzwerten für minimale, maximale und häufigste Dauer (analog zu PERT) ermittelt. Darin eingeflossen sind neben den reinen Bearbeitungszeiten auch Zeiten für Warenein- und -ausgang, Transport, Zwischenlagerung u.ä., also die vollständige Durchlaufzeit jedes Lieferanten.

Das Berechnungsmodell wurde um virtuelle Prozesse erweitert, um die Beauf-tragungszeitpunkte der Zulieferer abzubilden. Sie bewirken einen verzögerten Start des realen Vorgangs. Die Dauern der virtuellen Vorgänge werden zunächst so bemessen, dass dadurch im Mittel alle parallelen Vorgänge zeitgleich enden, der anschließende Montageprozess also (im Mittel) ohne Verzögerung beginnen kann.

Für die gesamte Supply Chain konnte mit dem vorgestellten numerischen Berechnungsverfahren die Durchlaufzeit unter Berücksichtigung der stochastischen Einflüsse auf die einzelnen Prozesse statistisch ermittelt werden. Der Vergleich mit anderen Verfahren ist in Abbildung 8 dargestellt. 


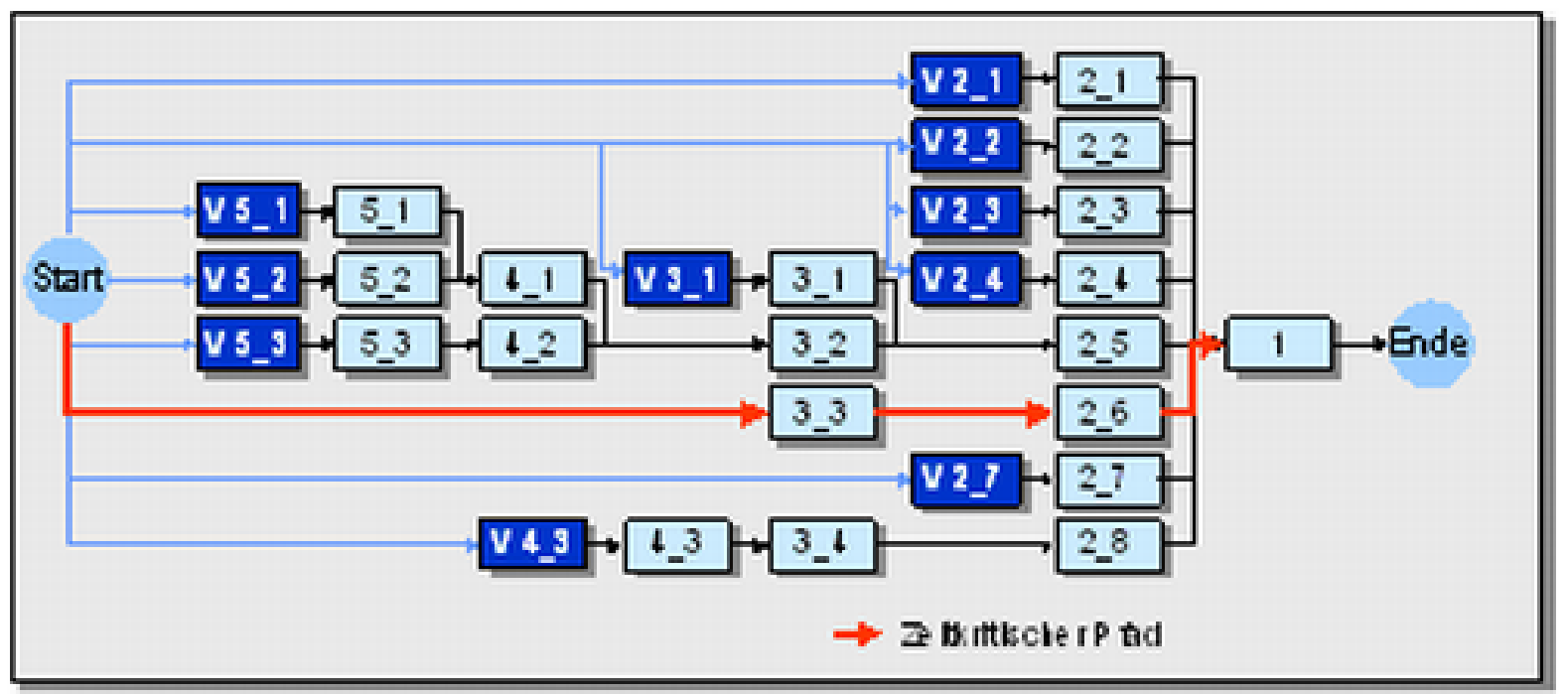

Abbildung 6: Struktur des Zulieferernetzwerks (hellblau) und Erweiterung um virtuelle Prozesse (dunkelblau)

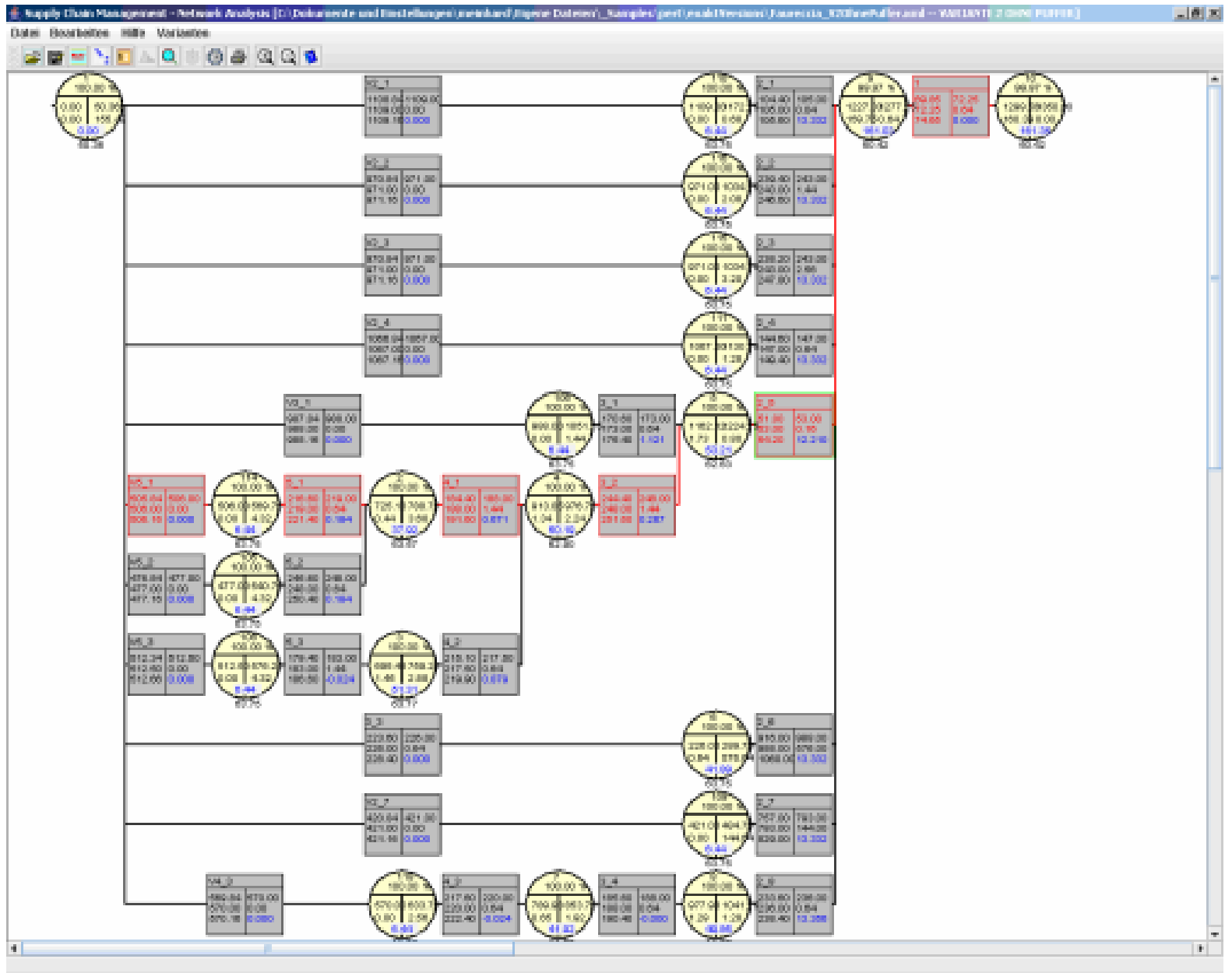

\section{Abbildung 7: Screenshot: Modell des Zulieferernetzwerks (rot: kritischer Pfad)}

Die Ergebnisse des numerischen Verfahrens decken sich dabei mit den durch Simulation erzielten, was als Nachweis für die Richtigkeit des Verfahrens angesehen werden kann. Im Vergleich dazu sind die mit PERT ermittelten Ergebnisse (mittlere Durchlaufzeit zu niedrig bei gleichzeitig zu großer Varianz) zu ungenau und bestätigen damit die oben dargelegten Schwächen der traditionellen Netzplantechnik.

Weiterhin besteht ein funktionaler Zusammenhang zwischen Durchlaufzeit und Liefertreue. Zur Veranschaulichung wird dabei die Wahrscheinlichkeit für die Überschreitung einer bestimmten Durchlaufzeit als Risiko für eine verspätete Lieferung aufgefasst (Abb. 9). Somit kann bei Vorgabe eines zulässigen Risikos die erforderliche Zeit vom Auftragsbeginn bis zur Lieferung (Liefertermin) bestimmt werden. 


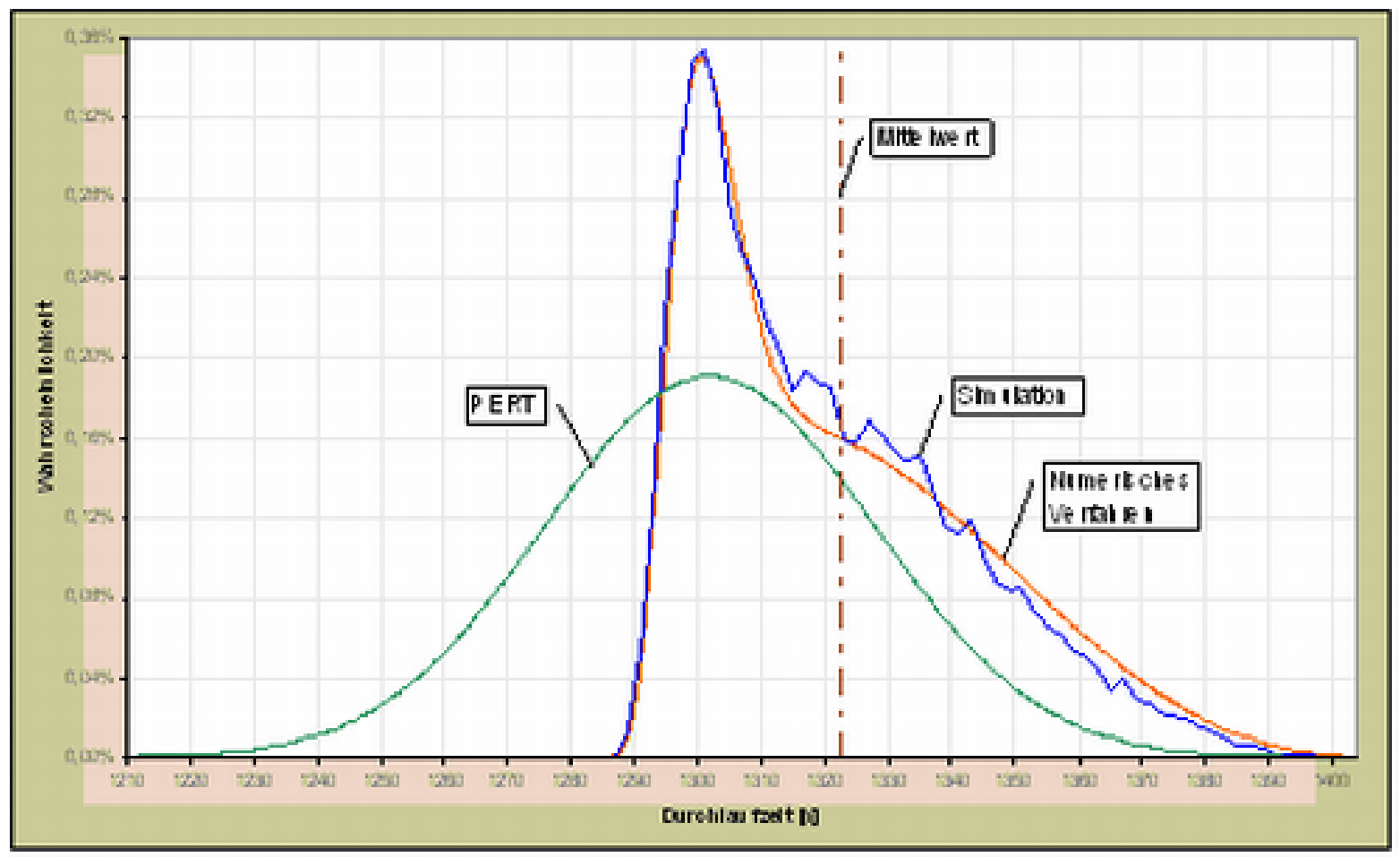

Abbildung 8: Vergleich der Ergebnisse zur Bestimmung der Durchlaufzeitverteilung

Durch Veränderung des Beauftragungszeitpunktes für einzelne Lieferanten lässt sich der Liefertermin verschieben. Eine frühere Beauftragung führt im Allgemeinen zur Verkürzung der Durchlaufzeit bei verändertem kritischen Weg im Netzplan. Dieser Zusammenhang ist an sich nicht neu, mit dem vorgestellten Verfahren kann die Auswirkung aber quantifiziert werden. Dabei kann bei jeweils gleichem „Aufwand“ (frühere Beauftragung um $x$ Zeiteinheiten für Prozess $j$ ) eine Verkürzung der Durchlaufzeit $\Delta t_{D L Z, j}=f(x, j)$ sowie eine Veränderung der mittleren, strukturbedingten Wartezeiten $\Sigma \mathrm{E}\left(\mathrm{t}_{\mathrm{wS}}\right.$, j) ermittelt werden. Für einen Variantenvergleich besteht damit eine Bewertungsmöglichkeit, inwiefern die verkürzte Durchlaufzeit möglicherweise durch höhere Lagerbestände erkauft wird. Unabhängig davon bleibt wegen der Schwankungen der Vorgangsdauern selbst ein gewisser Bestand zur Prozessentkopplung erforderlich. 


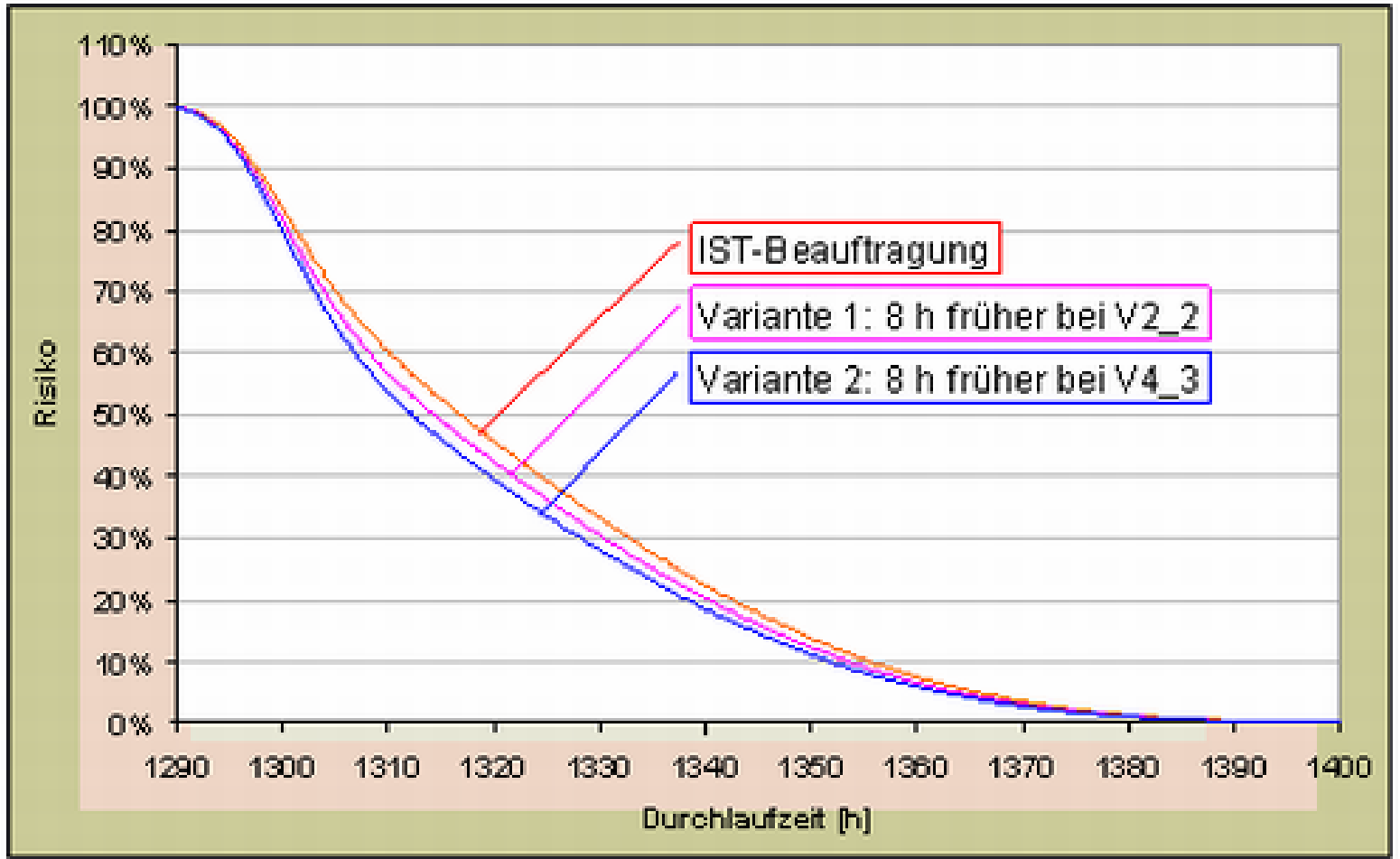

Abbildung 9: Auswirkung unterschiedlicher Beauftragungszeitpunkte auf die Liefertreue (dargestellt als Risiko für Terminüberschreitung)

\section{Zusammenfassung und Ausblick}

Mit dem vorgestellten numerischen Verfahren kann das Zeitverhalten in Supply Chains statistisch bestimmt und ausgewertet werden. Es liefert zur ereignisdiskreten Simulation gleichwertige Ergebnisse. Durch Änderung der Parameter der Vorgangsdauern lassen sich Auswirkungen auf die Gesamtdauer schnell und ohne spezielle Programmierkenntnisse (Simulationsexperte) ermitteln. Die berechnete strukturbedingte Wartezeit ist ein erstes Maß für mögliche Lagerbestände, die durch die Prozessverkettung entstehen.

Im Weiteren soll die Betrachtung zu Beständen entlang der Supply Chain vertieft werden. Die bei den vorgestellten Prozessverkettungen im Allgemeinen zunehmende Unsicherheit (Varianz) kann durch geeignete Entkopplungselemente (Lager, Puffer) wieder reduziert werden. Diese Elemente haben ABER EIN grundsätzlich anderes Verhalten als die bisher betrachteten Vorgänge: Ihr Zeitverhalten ist nicht vorgegeben, sondern resultiert aus den Schwankungen im vorgelagerten zeitlichen Ablauf und wirkt über den Pufferfüllgrad als dynamische Größe. Auch hier stellt sich die Frage nach der richtigen Dimensionierung, aber auch nach der richtigen Prozessintegration (Standort) derartiger Puffer.

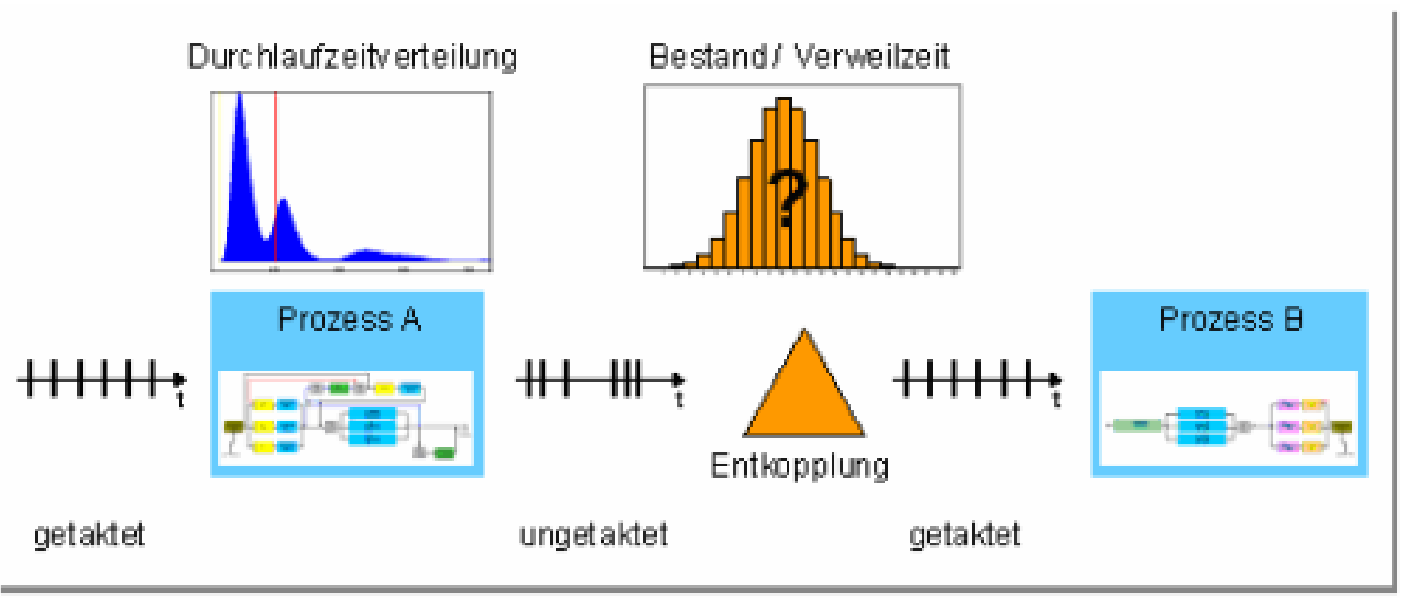

Abbildung 10: Puffer in einer Montagelinie zur Prozessentkopplung 
Abbildung 10 zeigt beispielhaft das Prinzip eines Entkopplungspuffers am Beispiel der Fahrzeugindustrie. Prozess A stellt dabei die Lackierung und Prozess B die Montagelinie dar. Beide Prozesse können selbst wieder aus einzelnen Unterprozessen bestehen. Ein getakteter Ankunftsstrom wird durch Prozess A auf Grund nicht konstanter Durchlaufzeiten in einen ungetakteten Strom transformiert. Für Prozess B ist aber erneut ein getakteter Strom erforderlich. Mit Hilfe eines Puffers gelingt es, die zeitlichen Schwankungen wieder auszugleichen, wobei die erforderliche Größe des Puffers ausschließlich von der berechneten Durchlaufzeitverteilung des vorgelagerten Prozesses abhängt.

Aus den statistischen Kenngrößen der Puffer-Belegung ist dann ein Rückschluss auf die Verteilung der Pufferaufenthaltszeit (Wartezeit) möglich, die nun wieder in die Durchlaufzeitbetrachtung zurückfließt und damit das Zeitverhalten sowie die daraus abgeleiteten Kenngrößen wie Mittelwert und Varianz für die Gesamtdauer sowie die Einhaltung des Liefertermins beeinflusst.

\section{Literatur}

[Croom00]

[DIN 69900]

[Erengüc99]

[Ioannou98]

[Malcolm59]

[Neumann75]

[Raghavan01]

[SCOR]

[Stadtler05]

[VDI 3633]
Croom, S., Romano, P., Giannakis, M.: Supply Chain Management: An analytical Framework for critical literature review. In: European Journal of Pur-chasing and Supply Management, 6 (2000) 1, S. 67 - 83

DIN 69900-1: Projektwirtschaft; Netzplantechnik; Begriffe. Ausgabe: 1987-08 Erengüç, S.S., Simpson, N.C., Vakharia, A.J.: Integrated production/distribution planning in supply chains: An invited review. In: European Journal of Operational Research, 115 (1999), S. $219-236$

Ioannou, P.G. und Martinez, J.C.: Project Scheduling using state-based proba-listic decision networks. Proceedings of the 1998 Winter Simulation Conference, S. 12871295, Washington D.C., Dec. 13.-16. 1998

Malcolm, D.G, Roseboom, J.H, Clark, C.E., Fazar, W.: Applications of a Technique for Research and Development Program Evaluation. In: Operations Research 7 (1959), S. 646-669

Neumann, K.: Operations-Research-Verfahren, Band III: Graphentheorie, Netzplantechnik. München: Hanser, 1975

Raghavan, N.R.S., Viswanadham, N.: Generalized Queueing Network Analysis of Integrated Supply Chains. In: International Journal of Production Research, 39 (2001) 2, S. $205-224$

Supply-Chain Operations Reference Model. Overview of SCOR-Version 6.1 https://acc.dau.mil/simplify/ev.php?ID=74835_201\&ID2=DO_TOPIC (Datum des letzten Zugriffs: 08.11.2005)

Stadtler, H.: Supply chain management and advanced planning - basics, over-view and challenges. In: European Journal of Operational Research, 163 (2005) 3, S. 575 $-588$

VDI-Richtlinie VDI 3633: Simulation von Logistik-, Materialfluss und Produktionssystemen, Blatt 1 Grundlagen, Dez. 2001 